\title{
Анализ напряженно-деформированного состояния стенки из титанового сплава ВТ6, полученной методом прямого лазерного выращивания
}

\author{
С. Ю. Иванов ${ }^{1}$, К. Д. Бабкин ${ }^{1}$, Е. В. Земляков ${ }^{1}$, И. К. Топалов ${ }^{1}$, Г. А. Туричин ${ }^{7}$, И. Д. Карпов ${ }^{2}$, В. Т. Эм² \\ ${ }^{1}$ Санкт-Петербургский государственный морской технический университет, www.smtu.ru, Санкт-Петербург \\ ${ }^{2}$ Национальный исследовательский щентр "Курчатовский институт", www.nrcki.ru, Москва
}

\begin{abstract}
Прямое лазерное выращивание является передовой технологией изготовления сложнопрофильных крупногабаритных изделий из широкого спектра сплавов, включающих сплавы на основе титана и никеля. В изготавливаемых изделиях в процессе выращивания формируется неравномерное поле остаточных напряжений и деформаций, которое оказывает существенное влияния на длительную прочность и работоспособность. Проведен анализ напряженно-деформированного состояния стенки из титанового сплава ВТ6, полученной методом прямого лазерного выращивания. В статье приведены результаты, выполненные расчетным методом конечных элементов и экспериментальным методом дифракции нейтронов. Установлено, что у краев наплавленной стенки вблизи подложки формируется область, где все три компоненты поля напряжений растягивающие. Более того, нормальные пластические деформации в данной области также растягивающие и достигают 2,5-3\%, а нормальные напряжения превышают предел текучести на 25\%. Продольные растягивающие напряжения близки к пределу текучести на всей длине нескольких последних слоев у вершины наплавки.
\end{abstract}

Ключевые слова: аддитивные технологии, прямое лазерное выращивание, титановый сплав, внутренние напряжения, остаточные напряжения, дифракция нейтронов

\section{Analysis of the Stress-Strain State of a Wall of VT6 Titanium Alloy Obtained by Direct Laser Deposition}

\author{
S. Yu. Ivanov', K. D. Babkin', E. V.Zemlyakov', I. K. Topalov', G.A. Turichin', I. D. Karpov², V. T. Em² \\ 1 St. Petersburg State Marine Technical University, www.smtu.ru, St. Petersburg \\ ${ }^{2}$ National Research Center "Kurchatov Institute", www.nrcki.ru, Moscow
}

Direct laser deposition is an advanced technology for the production of complex largesized products from a wide range of alloys, including alloys based on titanium and nickel. During the deposition process an uneven field of residual stresses and strains is formed in manufactured parts, which has a significant effect on long-term strength and performance. The analysis of the stress-strain state of the wall of VT6 titanium alloy obtained by direct laser deposition was carried out. The paper presents the results obtained numerically using finite element method and experimentally using neutron diffraction method. It was found 


\begin{abstract}
that at the edges of the deposited wall near the substrate, a region is formed where all three components of the stress field are tensile. Moreover, normal plastic strains in this area are also tensile and reach $2.5-3 \%$, and normal stresses exceed the yield strength by $25 \%$. Longitudinal tensile stresses are close to the yield strength along the entire length of the last few layers at the top of the buildup.
\end{abstract}

Keywords: additive technologies, direct laser deposition, titanium alloy, internal stresses, residual stresses, neutron diffraction

\section{ВВЕДЕНИЕ}

Прямое лазерное выращивание (ПЛв) является передовой технологией изготовления сложнопрофильных крупногабаритных изделий из широкого спектра сплавов, включающих сплавы на основе титана и никеля [1-6]. Основным преимуществом технологии является существенное сокращение производственного цикла за счет исключения из технологической цепочки операций сварки, литья и механической обработки. В процессе Плв небольшой объем изготавливаемого изделия претерпевает концентрированный и кратковременный нагрев до высоких температур. По мере перемещения источника теплоты нагреву подвергаются все новые объемы металла, а в ранее нагретых местах температура выравнивается. Таким образом, в выращиваемом изделии формируется неравномерное по объему температурное поле с большим температурным градиентом в области локального нагрева. Это вызывает в соседних участках изготавливаемого изделия разные по величине объемные изменения, которые ведут к появлению в металле внутренних усилий и образованию поля напряжений и деформаций. В отличие от температурного поля, которое исчезает после полного остывания конструкции, поле напряжений не исчезает, так как процесс его образования является необратимым. Поэтому после полного остывания в изделии имеются остаточные деформации и напряжения. Изучение процесса образования напряжений и деформаций имеет существенное значение для решения вопросов, связанных с проблемой технологической прочности и оценки работоспособности получаемых изделий.

В открытой печати опубликовано большое количество моделей и экспериментальных данных о распределении остаточных напряжений при селективном лазерном спекании [7, 8], прямом лазерном выращивании [9-14], дуговом выращивании $[15,16]$. Большая часть этих исследований сконцентрирована на экспериментальном определении остаточных напряжений методом дифракции нейтронов. При этом практически во всех случаях наплавка образцов производилась на гибкую подложку без остывания между слоями/проходами. Данные условия постановки эксперимента существенно отличаются от условий производства крупногабаритных изделий методом Плв, при которых подложка, как правило, жестко закреплена, а изготавливаемое изделие успевает остывать до достаточно низкой температуры перед выполнением каждого прохода / слоя.

Целью исследования является анализ кинетики напряженно-деформированного состояния стенки из титанового сплава ВТ6, полученной методом прямого лазерного выращивания.

\section{1. МЕТОДИКА ПРОВЕДЕНИЯ ИССЛЕДОВАНИЯ}

\section{1. Материал и параметры режима}

Прямое лазерное выращивание стенки из сплава ВТ6 осуществлялось на торце пластины толщиной 12 мм из сплава ВТ6 (рис. 1). Всего было наплавлено 50 слоев суммарной высотой 28 мм, каждый из которых включал 4 прохода высотой 0,56 мм. Экспериментально определенные механические свойства сплава ВТ6 при комнатной температуре были следующими: предел текучести 1100 МПа; модуль Юнга 111 ГПа. Установка для ПлВ включала волоконный лазер мощностью 5 кВт, технологическую головку с коаксиальной подачей порошка. Параметры процесса были следующими: мощность лазера 1,6 кВт; амплитуда осцилляции луча 1 мм; радиус луча 1 мм; скорость перемещения головки $30 \mathrm{~mm} / \mathrm{c}$. После выполнения каждого прохода наплавка охлаждалась за счет конвекции и теплопроводности до температуры 60-80 ㄷ․ Жесткая подложка и низкая межпроходная температура были выбраны для максимального приближения условий $\mathrm{K}$ реальным условиям изготовления крупногабаритных изделий. 

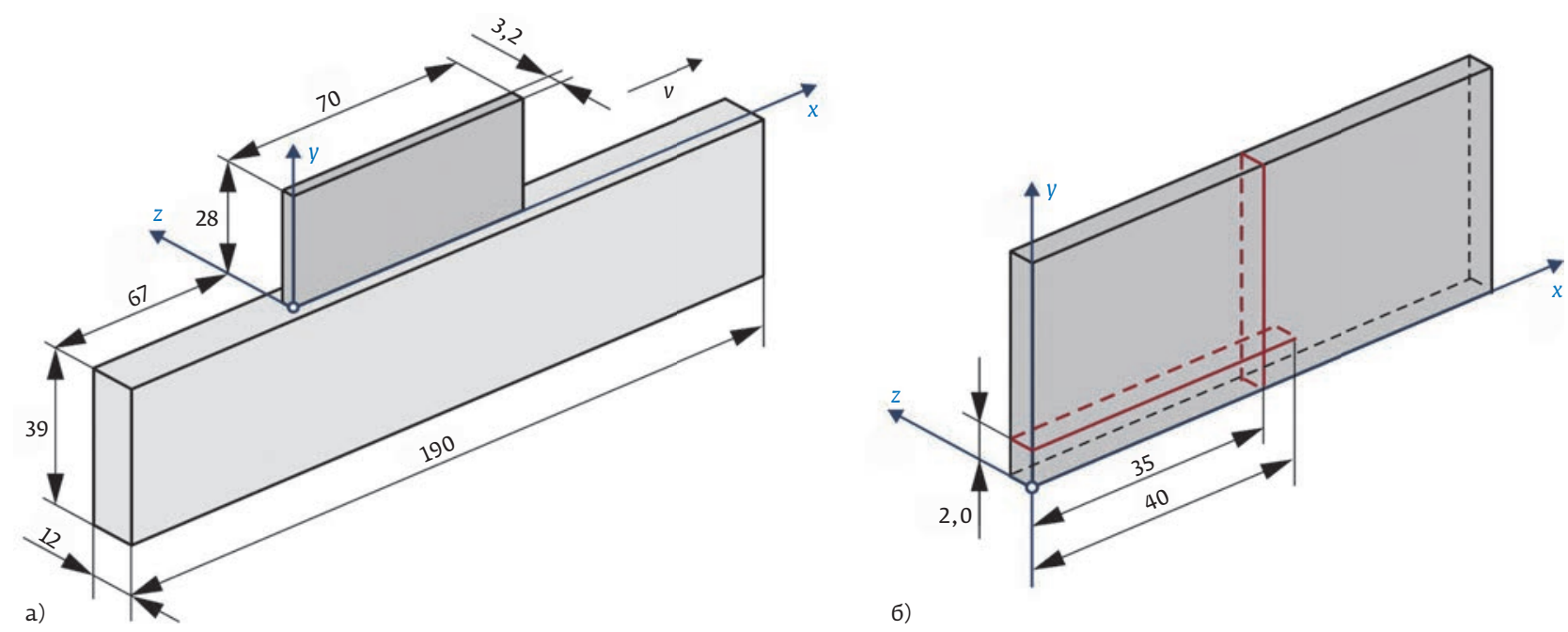

Puc. 1. (хема эксперимента: а) схема наплавленной стенки и выбранная система координат; б) положение горизонтального и вертикального сечений в которых проводился замер остаточных напряжений

\section{2. Методика экспериментального определения остаточных напряжений}

Измерения проводились на дифрактометре СТРЕСС, установленном на горизонтальном экспериментальном канале ГЭК-3 реактора ИР-8. Особенностью данной установки является то, что используется двойной монохроматор PG002/Si220 из пиролитического графита и фокусирующего кристалла кремния [17], который выводит монохроматический пучок нейтронов с длиной волны $\lambda=1,56 \AA$. Для измерения напряжений использовался дифракционный пик (103) титана на угле рассеяния $2 \theta \approx 71,40$. Пробный объем $\sim 7$ мм $^{3}$ $\left(1,5 \times 1,5 \times 3\right.$ мм $\left.^{3}\right)$ определялся кадмиевыми щелями шириной 1,5 мм и высотой 3 мм.

Нейтрон-дифракционный метод измерения внутренних напряжений основан на измерении разницы между межплоскостным расстоянием $d_{h k l}$ плоскостей $(h k l)$ в кристаллической решетке напряженного материала и расстоянием $d_{0, h k l}$ в ненапряженной решетке. Определение межплоскостных расстояний основано на законе дифракции Брэгга (для простоты далее миллеровские индексы плоскостей $h, k, l$ опущены):

$$
2 d \sin \theta=n \lambda,
$$

где $d$ - межплоскостное расстояние, $\theta$ - брэгговский угол рассеяния, $n$ - целое число, $\lambda$ - длина волны нейтронов.

На дифрактометре с постоянной длиной волны $\lambda$ межплоскостному расстоянию $d$ соответствует ди $\phi_{-}$ ракционный пик на угле рассеяния $2 \theta$ так, что относительную деформацию можно определить по сдвигу брэгговского угла рассеяния для напряженного образца относительно ненапряженного образца [18]:

$$
\varepsilon=\frac{d-d_{0}}{d_{0}}=-\left(2 \theta-2 \theta_{0}\right) \frac{\operatorname{ctg} \theta_{0}}{2} .
$$

С помощью щелей в кадмиевых экранах (кадмий сильно поглощает тепловые нейтроны), установленных в падающем и дифрагированных пучках нейтронов, выделяется пробный объем или "gauge volume» ( 1-100 мм³) на оси дифрактометра и измеряется дифракционный пик от маленькой части массивного образца, находящейся в пробном объеме. Обычно в пробном объеме содержится большое число кристаллитов $\left(\sim 10^{7}\right)$, которые статистически ориентированы по направлениям. Поэтому при любой ориентации образца в пробном объеме найдется достаточно большое количество кристаллитов, которые ориентированы так, что плоскости (hkl) в них удовлетворяют условиям закона Брэгга, и они дают вклад в дифракционный пик. Поскольку измеряется дифракционный пик нейтронов, отраженных пробным объемом, то определяется деформация, усредненная по этому объему. Совмещая точки образца, в которых нужно измерить напряжения, с центром пробного объема можно измерить распределение деформаций по образцу.

Измеряются компоненты деформации $\varepsilon_{x}, \varepsilon_{y}, \varepsilon_{z}$ вдоль трех взаимно перпендикулярных главных направлений $x, y, z$ в образце и, пользуясь обоб- 
щенным законом Гука, вычисляют компоненты напряжений $\sigma_{x}, \sigma_{y}, \sigma_{z}$ вдоль этих направлений:

$$
\sigma_{i}=\frac{E}{(1+v)(1-2 v)}\left[(1-2 v) \varepsilon_{i}+v\left(\varepsilon_{x}+\varepsilon_{y}+\varepsilon_{z}\right)\right]
$$

где $i=x, y$ или $z ;$ E - модуль Юнга; v - коэффициент Пуассона.

\section{3. Расчетная методика определения напряженно-деформированного состояния}

В современных подходах моделирования термомеханических процессов традиционным является подход моделирования напряженно-деформированного состояния I рода на основе феноменологических моделей, когда в произвольно достаточно малом элементарном объеме тензоры напряжений $\sigma_{i j}$ и деформаций $\varepsilon_{i j}(i, j)=x, y, z$ усреднены характеристиками напряженно-деформированного состояния для данного объема с пространственными координатами $x, y, z$ в момент времени $t$. Тензоры $\sigma_{i j}$ и $\varepsilon_{i j}$ соподчиняются классическим законам механики деформируемой сплошной среды (уравнения движения либо равновесия, уравнения связи между компонентами тензора $\varepsilon_{i j}$ и вектора перемещений $\left.u_{i}\right)$. Основные особенности технологического воздействия отражаются в граничных условиях и уравнениях связи между тензорами $\varepsilon_{i j}$ и $\sigma_{i j}$. Рассматриваемая задача является нелинейной и может быть решена только численными методами, наиболее широко применяемым из которых на данный момент является метод конечных элементов (МКЭ).
Моделирование процесса Плв существенно осложнено тем, что локальный высокотемпературный нагрев концентрированным источником теплоты требует построения достаточно мелкой сетки в области его действия. Получаемая расчетная модель может содержать сотни тысяч и даже миллионы элементов, что требует существенных вычислительных мощностей для выполнения расчетных операций. При этом для определения остаточных напряжении и деформаций необходимо проследить всю историю нагружения конструкции, то есть учесть нагрузку, создаваемую последовательно всеми наплавляемыми проходами, суммарная длина которых может достигать многих километров. Процесс расчетного определения напряженно-деформированного состояния изделия в процессе ПЛВ состоит в последовательном решении связанных задач теплопроводности и термоупругопластичности. Все временные промежутки между проходами разбиваются на временные шаги. Таким образом прослеживается вся кинетика изменений температуры, напряжений и деформаций.

Процесс последовательного выращивания изделия учитывался с помощью следующего искусственного приема: в тех конечных элементах, в которых в текущий момент отсутствует наплавленный металл, задавались заниженные в $10^{4}$ раз теплопроводность, энтальпия и модуль упругости (рис. 2а). Ввод энергии при наплавке проходов моделировался подвижным объемным источником теплоты с тепловой эффективностью равной 0,4 [19]. При решении учитывали температурные зависимости теплофизических и механических свойств

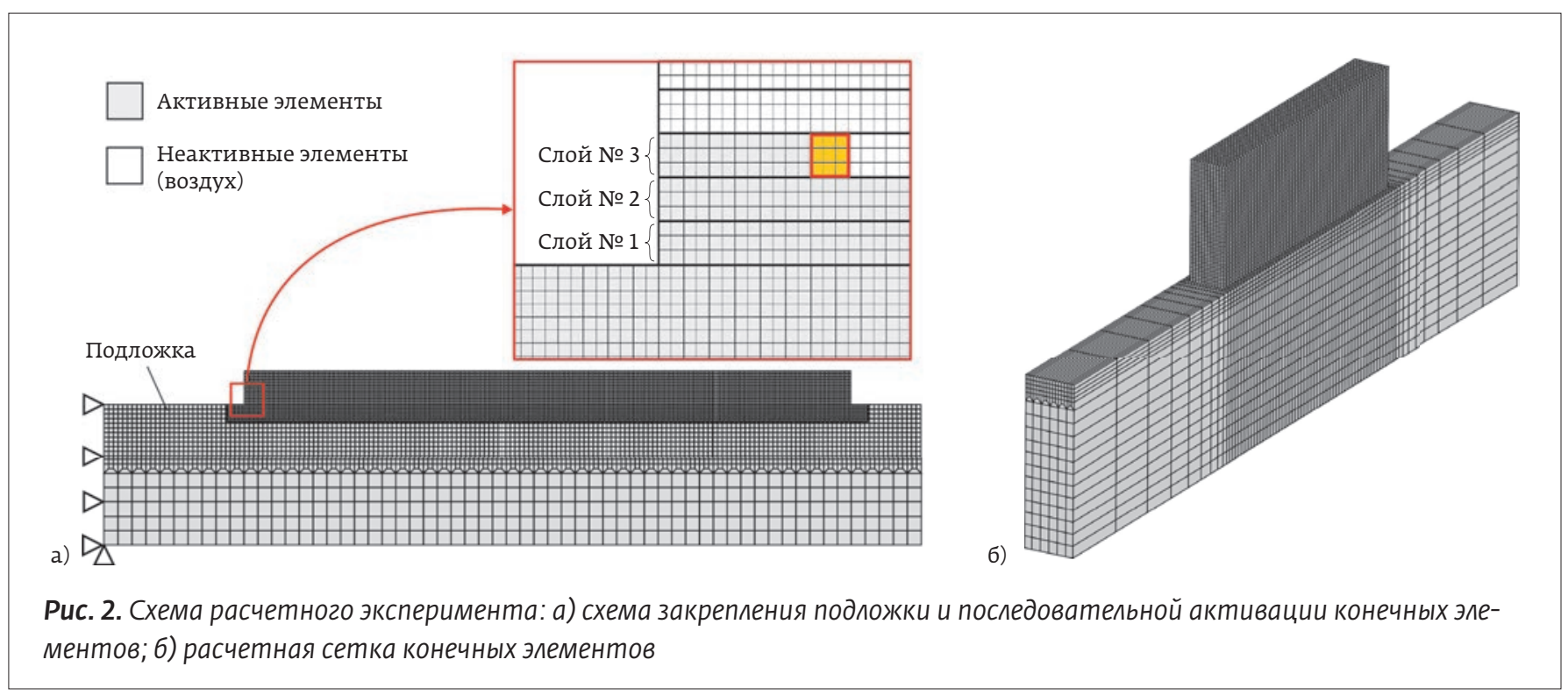


материала [20, 21]. Использовали 8-узловые трехмерные изопараметрические конечные элементы со сгущением в зоне наплавляемых слоев, где высокий температурный градиент (рис. 2б).

\section{2. РЕЗУЛЬТАТЫ ИССЛЕДОВАНИЙ И ИХ ОБСУЖДЕНИЕ}

Экспериментально замеренное распределение остаточных напряжений показано на рис. 3. Распределение значений межплоскостного расстояния ненапряженной решетки $d_{0}$ были определены из предположения, что напряжения по толщине наплавки близки к нулю, т.е. использована схема плоского напряженного состояния. Рассчитан ные методом конечных элементов результаты усреднены по толщине наплавленной стенки на базе, соответствующей размеру пробного объема, использованного при экспериментальном определении напряжений. Видно, что экспериментальные и расчетные результаты удовлетворительно совпадают. Расхождение наблюдается вблизи края наплавки на расстоянии менее 10 мм в горизонтальном сечении (рис. 3а). Согласно расчетным результатам в этой области поперечные напряжения не равны нулю. В центральном сечении ( $x=35$ мм) удовлетворительное совладение расчетных и экспериментальных значений наблюдается лишь вблизи подлодки. Увеличение ошибки при удалении от подложки может быть связано с тем, что расчетная модель не учитывала релаксацию напряжений. Следует отметить, что характер изменения расчетных и экспериментальных кривых одинаковый.
Распределение расчетных продольных $\left(\sigma_{x}\right)$, нормальных $\left(\sigma_{y}\right)$ и поперечных $\left(\sigma_{z}\right)$ остаточных напряжений в наплавленной стенке показаны на рис. 4а-в. Представленные результаты усреднены по толщине наплавленной стенки на базе, соответствующей размеру пробного объема, использованного при экспериментальном определении напряжений. Распределение всех компонент поля напряжений практически симметрично относительно центрального сечения наплавки, соответствующего координате $x=35$ мм. Можно выделить несколько областей концентрации растягивающих напряжений, значение которых близко или превышает предел текучести наплавляемого материала при комнатной температуре. Продольные растягивающие напряжения концентрируются практически на всей длине последних слоев у вершины наплавки. Механизм их формирования следующий: в процессе нагрева узкий участок наплавки, включающий наплавляемый слой и зону термического влияния (ЗТВ), стремится увеличить свой объем вследствие температурного расширения. Поскольку окружающий холодный металл имеет высокую жесткость и препятствует расширению, в нагреваемой области возникают временные сжимающие напряжения и пластические деформации. Выравнивание температуры по сечению наплавки и подложки в процессе охлаждения приводит к перераспределению температурных деформаций. Участки металла, подвергшиеся пластическому деформированию в процессе нагрева, не могут свободно укорачиваться ввиду их взаимосвязи с остальными участками наплавки. Это приводит к тому, что сжимающие напряжения сменяются рас-
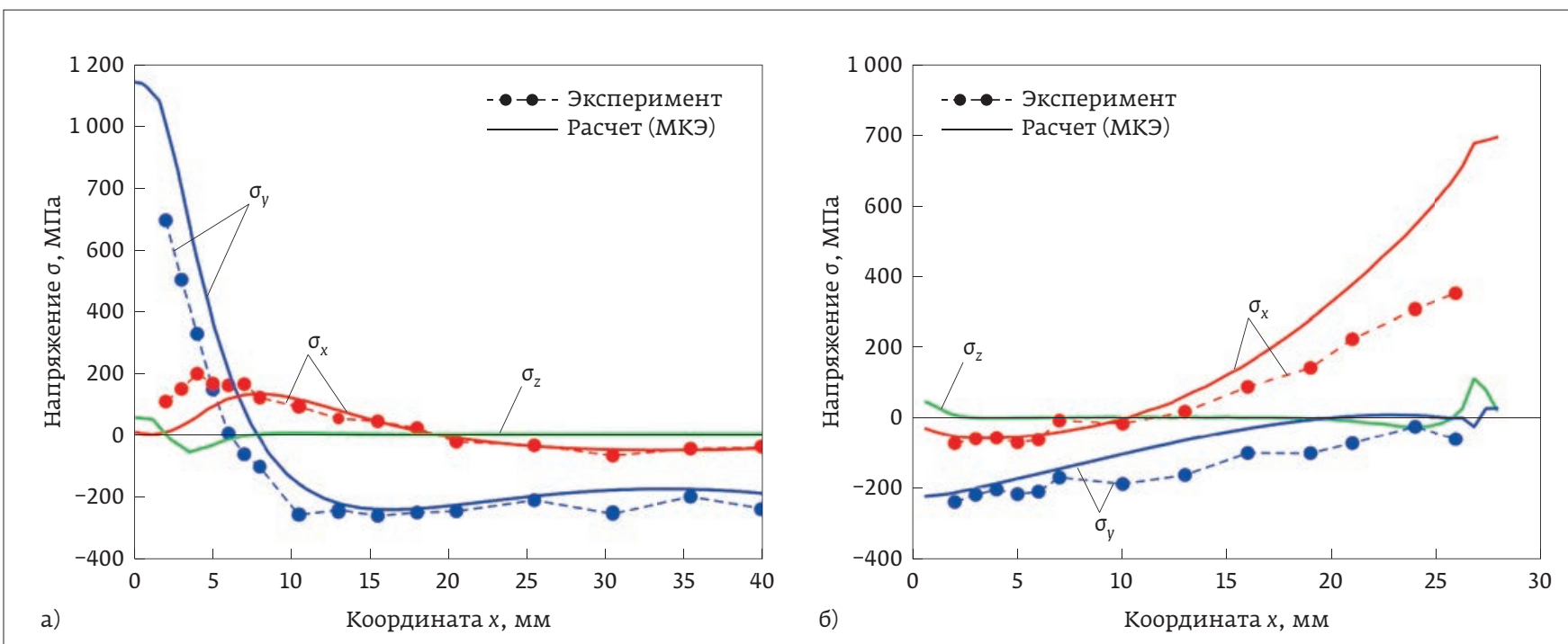

Puc. 3. Распределение остаточных напряжений (см. рис. 16): а) в горизонтальном сечении; б) в вертикальном сечении 
тягивающими, а сжимающие временные пластические деформации частично компенсируются растягивающими пластическими деформациями. Также область повышенных продольных напряжений наблюдается у подложки вблизи краев наплавки. Наибольшие растягивающие напряжения, соответствующие нормальной компоненте $\sigma_{y}$, достигаются на боковых краях наплавленной стенки. Анализ их изменения в процессе наплавки показал, что по мере увеличения высоты наплавки размер этой области постепенной увеличивается вследствие увеличения изгибающего момента. Следует отметить, что напряжения в данной области превышают предел текучести более чем на 25\%. Анализ нормальных остаточных пластических деформаций (рис. 4г-д) показал, что в этой области они - растягивавшие. При этом они достигают максимального значения в первых трех-четырех наплавленных проходах, как видно на их распределении в сечении $x=2,5$ мм (рис. 4д). Наименьшее значение из всех компонент поля остаточных напряжений достигают попереч- ные напряжения, поскольку толщина наплавки мала. Их величина лишь в очень небольшой области превышает 150 МПа.

Анализ поля остаточных напряжений показал, что у краев наплавленной стенки вблизи подложки формируется область, где все три компоненты поля напряжений - растягивающие. Более того, нормальные пластические деформации в данной области также растягивающие и достигают 3\%, а нормальные напряжения превышают предел текучести на 25\%. Механические свойства сплава ВТ6, полученные методом ПЛВ, имеют ярко выраженную анизотропию и существенно зависят от параметров технологического процесса и качества исходного порошка. Анализ литературных данных показал, что пластичность сплава ВТ6 колеблется в пределах 4-12\% в продольном направлении и 0,8-6\% в нормальном [22]. Из вышесказанного можно сделать вывод, что области и в начале, и конце наплавленной стенки вблизи подложки потенциально склонны к возникновению разрушения.

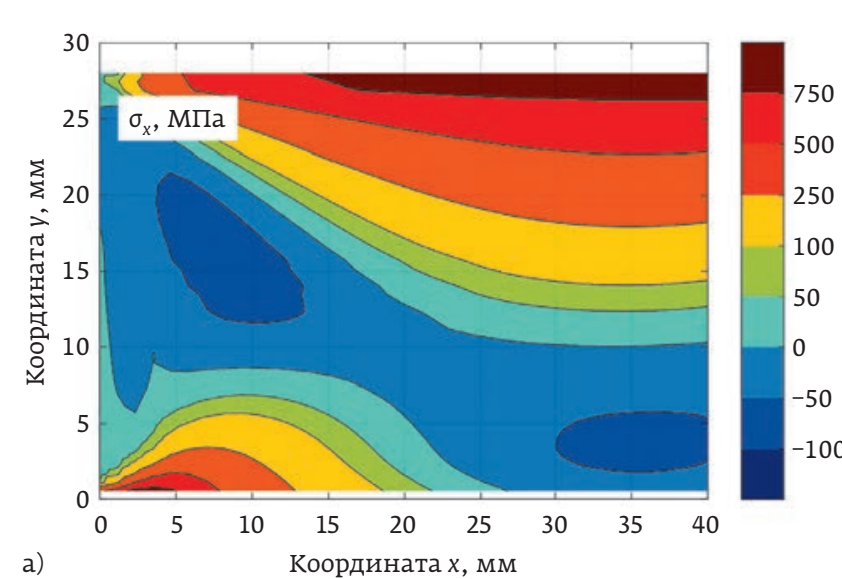

a)

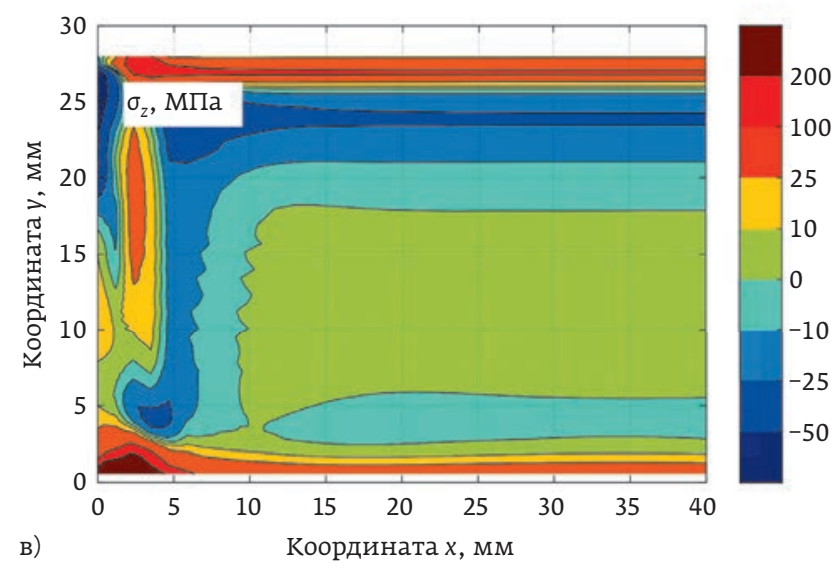

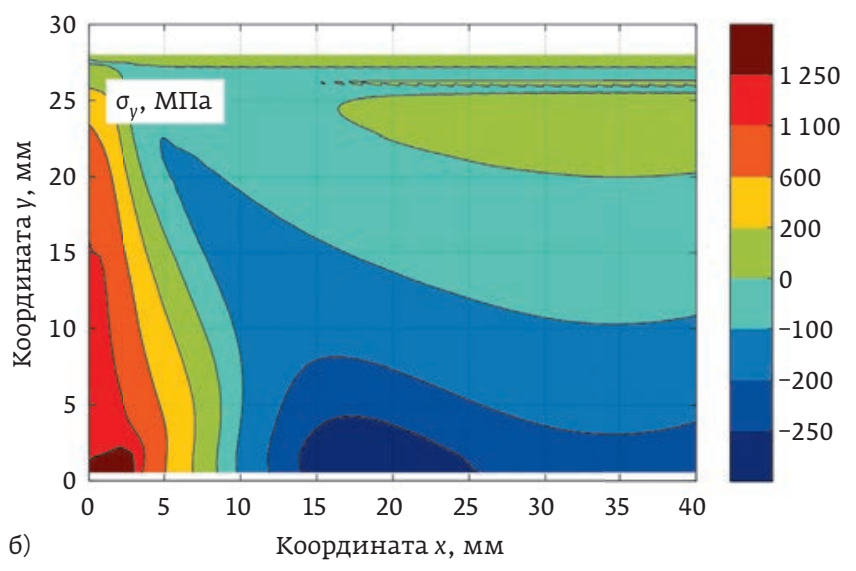
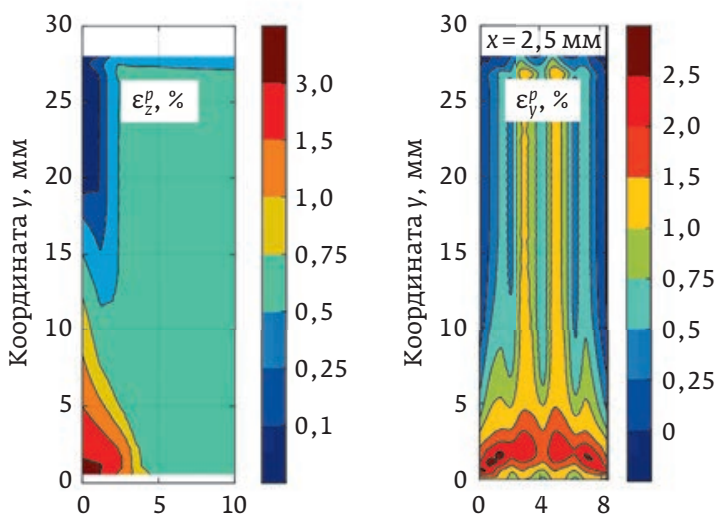

д) Координата z, мм

Puc. 4. Распределение расчетных напряжений в наплавленной стенке: $a)$ - в) остаточные продольные ( $\left.\sigma_{x}\right)$, нормальные $\left(\sigma_{y}\right)$ и поперечные $\left(\sigma_{z}\right)$ напряжения; 2), д) нормальные пластические деформации 


\section{ВЫВОДЫ}

На основе анализа экспериментально замеренного распределения остаточных напряжений и результатов расчетного анализа кинетики напряженнодеформированного состояния наплавки в процессе прямого лазерного выращивания можно сделать следующие выводы:

- У краев наплавленной стенки вблизи подложки формируется область, где все три компоненты поля напряжений - растягивающие. Более того, нормальные пластические деформации в данной области также растягивающие и достигают 2,5-3\%, а нормальные напряжения превышают предел текучести на $25 \%$.

- Продольные растягивающие напряжения близки к пределу текучести на всей длине нескольких последних слоев у вершины наплавки.

- Рассчитанные методом конечных элементов остаточные напряжения удовлетворительно совпадают с экспериментально замеренными методом дифракции нейтронов.

\section{БЛАГОДАРНОСТЬ}

Работа выполнена в ходе реализации комплексного проекта при финансовой поддержке Правительства Российской Федерации (Соглашение с Минобрнауки России № 075-11-2018-067 от “28» апреля 2017 года (внут. № 03.G25.31.0240)).

\section{ВКЛАД ЧЛЕНОВ АВТОРСКОГО КОЛЛЕКТИВА В РАБОТУ}

Идея проведения эксперимента, организация и руководство проектом: Г.А.Туричин, Е.В.Земляков; дизайн и проведение эксперимента по изготовлению образцов: К.Д. Бабкин, И.К. Топалов; экспериментальное определение остаточных напряжений: .Д. Карпов, В.Т.Эм; численное моделирование, обобщение данных и обработка результатов: С. Ю.Иванов, И. К. Топалов. Все авторы приняли участие в написании статьи и дополнили рукопись в части своей работы.

\section{СПИСОК ЛИТЕРААТУРЫ}

1. Туричин Г.А., Земляков Е. В., Климова О. Г., Бабкин К. Д., Шамрай Ф.А. Колодяжный Д. Ю. Прямое лазерное выращивание - перспективная аддитивная технология для авиадвигателестроения. Сварка и Диагностика. 2015; 3: 54-57.

Turichin G. A., Zemlyakov E. V., Klimova O. G., Babkin K. D., SHamraj F.A., Kolodyazhnyj D.YU. Pryamoe lazernoe vyrashchivanie - perspektivnaya additivnaya tekhnologiya dlya aviadvigatelestroeniya. Svarka i Diagnostika. 2015; 3: 54-57.

2. Агеев Р. В., Кондратов Д. В., Маслов Ю. В. Применение аддитивных технологий при проектировании и производстве деталей аэрокосмических объектов. Полет. 2013; 6: 35-39.
Ageev R. V., Kondratov D. V., Maslov YU.V. Primenenie additivnyh tekhnologij pri proektirovanii i proizvodstve detalej aerokosmicheskih ob»ektov. Polet. 2013; 6: 35-39.

3. Дорохов А. Ф., Абачараев М. М. Аддитивные технологии в производстве корабельной энергетики. Вестник Астраханского государственного технического университета. Сер. Морская техника и технология. 2015; 2: 42-47. Dorohov A. F., Abacharaev M. M. Additivnye tekhnologii v proizvodstve korabel'noj energetiki. Vestnik Astrahanskogo gosudarstvennogo tekhnicheskogo universiteta. Ser. Morskaya tekhnika i tekhnologiya. 2015; 2: 42-47.

4. Debroy T., Wei H. L., Zuback J. S., Mukherjee T., Elmer J. W., Milewski J. 0. Allison Michelle Beese, A. Wilson-Heid, A. De, W. Zhang. Additive manufacturing of metallic components - process, structure and properties. Progress in Materials Science. 2018; (92): 112-224.

5. Gu D. D., Meiners W., Wissenbach K., Poprawe R. Laser additive manufacturing of metallic components: materials, processes and mechanisms. International materials reviews. 2012; 57(3): 133-164.

6. Liu S., Shin Y. C. Additive manufacturing of Ti6Al4V alloy: A review. Materials \& Design. 2019; 164: 107552.

7. Nadammal N., Cabeza S., Mishurova T., Thiede T., Kromm A., Seyfert C., Farahbod L., Haberland C., Schneider J. A., Dolabella Portella P., Bruno G. Effect of hatch length on the development of microstructure, texture and residual stresses in selective laser melted superalloy Inconel 718. Materials \& Design. 2017;134:139-150.

8. Thiede T., Cabeza S., Mishurova T., Nadammal N. Residual stress in selective laser melted inconel 718: Influence of the removal from base plate and deposition hatch length. Materials Performance and Characterization. 2018; 7(4):1-20.

9. Mukherjee T., Zhang W., DebRoy T. An improved prediction of residual stresses and distortion in additive manufacturing. Computational Materials Science. 2017; (126): 360-372.

10. Wang Z., Denlinger E., Michaleris P., Stoica A. D., Ma D., Beese M. Residual stress mapping in Inconel 625 fabricated through additive manufacturing: Method for neutron diffraction measurements to validate thermomechanical model predictions. Materials \& Design. 2017; (113): 169-177.

11. Bayerlein F., Bodensteiner F., Zeller C., Hofmann M., Zaeh M. F. Transient development of residual stresses in laser beam melting - A neutron diffraction study. Additive Manufacturing. 2018; (24): 587-594.

12. Wang Z., Denlinger E., Michaleris P., Stoica A. D., Ma D., Beese A. M. Residual stress mapping in Inconel 625 fabricated through additive manufacturing: Method for neutron diffraction measurements to validate thermomechanical model predictions. Mater Des. 2017; 113169-113177.

13. Sochalski-Kolbus L., Payzant E. A., Cornwell P. A., Watkins T. R., Babu S. S., Dehoff R. R., Lorenz M., Ovchinnikova O., Duty C. Comparison of residual stresses in Inconel 718 simple parts made by electron beam melting and direct laser metal sintering. Metall Mater Trans A. 2015; 46(3): 1419-1432.

14. Luzin V., Hoye $\mathbf{N}$. Stress in thin wall structures made by layer additive manufacturing. Materials Research Proceedings. 2016; 2: 497-502.

15. Szost B. A., Terzi S., Martina F., Boisselier D., Prytuliak A., Pirling T., Hofmann M., Jarvis D. J. A comparative study of additive manufacturing techniques: Residual stress and microstructural analysis of CLAD and WAAM printed Ti-6Al-4V components. Materials \& Design.2016; 89: 559-567.

16. Martina F., Roy M. J., Szost B. A., Terzi S., Colegrove P. A., Williams S. W., Withers P. J. Meyer J., Hofmann M. Residual stress of as-deposited and rolled wire+arc additive manufacturing Ti-6Al-4V components. Materials Science and Technology. 2016; 32(14): 1439-1448.

17. Em V.T., Balagurov A. M., Glazkov V. P., Karpov I. D., Mikula P., Miron N. F., Somenkov V. A., Sumin V.V. A double-crystal monochromator for neutron stress diffractometry, Instruments and Experimental Techniques. 2017; 60 (4): 526-532.

18. Hutchings M. T., Withers P. J., Holden T. M. Introduction to the Characterization of Residual Stress by Neutron Diffraction. - London: Taylor and Francis. 2005.

19. Kwon H., Baek W. K., Kim M. S., Shin W. S., Yoh J. J. Temperature-dependent absorptance of painted aluminum, stainless steel 304 , and titanium for $1.07 \mathrm{Im}$ and $10.6 \mathrm{Im}$ laser beams. Opt. Lasers Eng. 2012; 50:114-121.

20. Cao J., Gharghouri M., Nash P. Finite-element analysis and experimental validation of thermal residual stress and distortion in electron beam additive manufactured Ti-6Al-4V build plates. Journal of Materials Processing Technology. 2016; 237: 409-419.

21. MSC Software. Simufact Additive. Material Database. 2016

22. Lewandowski J.J., Seifi M. Metal additive manufacturing: A review of mechanical properties. Annual Review of Materials Research. 2016; 46(1): 151-186. 